\title{
Long-term clinical outcome of the Charité III total lumbar disc replacement
}

\author{
J. Kitzen ${ }^{1}$ - M. G. M. Schotanus ${ }^{2}$ - S. M. J. van Kuijk ${ }^{3}$ - E. M. C. Jutten ${ }^{1}$ - N. P. Kort ${ }^{2}$ - L. W. van Rhijn ${ }^{1}$ - P. C. Willems ${ }^{1}$
}

Received: 25 May 2019 / Revised: 30 November 2019 / Accepted: 18 January 2020 / Published online: 29 January 2020

(c) The Author(s) 2020

\begin{abstract}
Purpose To compare the long-term clinical results and complications of two revision strategies for patients with failed total disc replacements (TDRs).

Methods In 19 patients, the TDR was removed and the intervertebral defect was filled with a femoral head bone strut graft. In addition, instrumented posterolateral fusion was performed (removal group). In 36 patients, only a posterolateral instrumented fusion was performed (fusion group). Visual analogue scale (VAS) for pain and Oswestry Disability Index (ODI) were completed pre- and post-revision surgery. Intra- and post-operative complications of both revision strategies were assessed. Results The median follow-up was 12.3 years (range 5.3-24.3). In both the removal and fusion group, a similar $(p=0.515$ and $p=0419$, respectively) but significant decrease in VAS- ( $p=0.001$ and $p=0.001$, respectively) and ODI-score $(p=0.033$ and $p=0.013$, respectively) at post-revision surgery compared to pre-revision surgery was seen. A clinically relevant improvement in VAS- and ODI-score was found in $62.5 \%$ and $43.8 \%$ in the removal group and in $43.5 \%$ and $39.1 \%$ in the fusion group ( $p=0.242$ and $p=0.773$, respectively). Removal of the TDR was associated with substantial intra-operative complications such as major vessel bleeding and ureter lesion. The percentage of late reoperations for complications such as pseudarthrosis was comparable for both revision strategies.

Conclusions Revision of a failed TDR is clinically beneficial in about half of the patients. No clear benefits for additional TDR removal as compared to posterolateral instrumented fusion alone could be identified. Particularly, when considering the substantial risks and complications, great caution is warranted with removal of the TDR.
\end{abstract}

\section{Graphic abstract}

These slides can be retrieved under Electronic Supplementary Material.
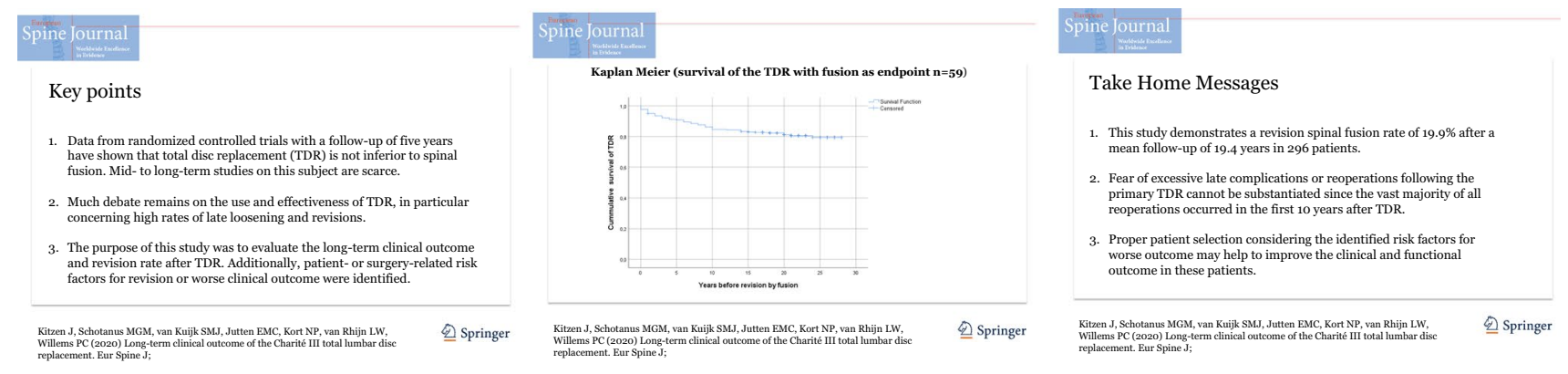

Keywords Degenerative disc disease $\cdot$ Lumbar spine $\cdot$ Clinical follow-up $\cdot$ Spine arthroplasty $\cdot$ Risk factors

Electronic supplementary material The online version of this article (https://doi.org/10.1007/s00586-020-06308-8) contains supplementary material, which is available to authorized users.

J. Kitzen

j.kitzen@mumc.nl

Extended author information available on the last page of the article

\section{Introduction}

Fusion of a symptomatic lumbar spinal motion segment is still considered the gold standard for operative treatment of patients with degenerative disc disease (DDD) not 
responding to conservative care [1-3]. However, spinal fusion is associated with side effects such as cranial facet joint violations, decrease in sagittal motion, pseudarthrosis and symptomatic adjacent-level disease [4-7].

Total lumbar disc replacement (TDR) has been introduced to avoid those fusion-related side effects based on the hypothesis that chronic low back pain (CLBP) originates from DDD. However, TDR has also been associated with drawbacks, such as subsidence, luxation or malposition of the implant, increasing axial rotational instability and excessive loads to the facet joints [8-10]. In a meta-analysis, an average reoperation rate of $7.8 \%$ at 2 - to 5 -year follow-up was found [9]. Several studies with midto long-term results reported that $6-14 \%$ of the patients had revision fusion surgery after TDR [11-17].

Data from randomized controlled trials with a followup of 5 years have shown that TDR is not inferior to spinal fusion [1-3]. Nonetheless, much debate remains on the use and effectiveness of TDR, in particular concerning high rates of late loosening and revisions [18]. Mid- to long-term studies on this subject are scarce $[2,3,11-17$, 19]. Only six studies have a mean follow-up of 10 years or more [11-14, 16, 17].

The purpose of this study was to evaluate the longterm clinical and functional outcome in terms of patient satisfaction and complication and revision rate after TDR. Additionally, an assessment was made to identify patientor surgery-related risk factors for revision or worse clinical outcome.

\section{Materials and methods}

\section{Patient selection}

The current study was approved by the Medical Ethics Committee METC Z (16-N-22) and registered at the Dutch Trial Registry (NTR5710). The medical records of all patients who had undergone a TDR by a single surgeon using a SB Charité III between 1989 and 2000 at the Zuyderland Medical Centre, Sittard-Geleen, the Netherlands, were reviewed. Altogether, 405 consecutive patients were identified.

TDR had been performed as treatment for patients with predominantly axial low back pain with failure of appropriate conservative measures and the presence of lumbar DDD as determined by plain radiographs and/or MRI. Preoperatively, all patients had undergone fluoroscopically guided provocation discography to rule out non-discogenic pain sources. No facet joint injections were performed. Radiculopathy, spondylolisthesis or spondylolysis were considered a contraindication for TDR. Patients with a previous discectomy or small decompression were not excluded in this study (Table 1). Complications were recorded when a reoperation needed to be performed. Revision surgery by spinal fusion of the TDR was defined as a failure.

\section{Clinical and subjective outcome evaluation}

Patient-reported outcome measures (PROMs) were obtained in all TDR patients who had not been revised at latest followup $(n=237)$. Back- and leg-pain intensity was recorded with a visual analogue scale (VAS, $0-10,10$ being 'worst pain').
Table 1 Summary of subgroup patient demographic and surgical data

\begin{tabular}{llll}
\hline & No revision & Revision & $p$ value $^{\mathrm{a}}$ \\
\hline$N(\%)$ & $237(80.1)$ & $59(19.9)$ & \\
Follow-up in years (range) & $19.5(13.7-25.6)$ & $19.1(7.6-26.3)$ & \\
Males, number (\%) & $102(84.3)$ & $19(15.7)$ & 0.130 \\
Mean age at time of surgery, years (range) & $41.9(22.0-60.0)$ & $40.6(22.0-63.0)$ & $0.227^{\mathrm{b}}$ \\
Surgical levels & & & \\
L2-L3 (\%) & $1(33.3)$ & $2(66.7)$ & $\mathbf{0 . 0 4 2}$ \\
L3-L4 (\%) & $14(73.7)$ & $5(26.3)$ & 0.472 \\
L4-L5 (\%) & $120(77.9)$ & $34(22.1)$ & 0.336 \\
L5-S1 (\%) & $146(79.8)$ & $37(20.2)$ & 0.875 \\
Number of levels (one: two: three) & $193-44-0$ & $43-14-2$ & $\mathbf{0 . 0 1 1}$ \\
Indication for lumbar disc replacement $(n=261)$ & & & \\
DDD without any other accompanying pathologies $(\%)$ & $160(76.6)$ & $49(23.4)$ & 0.221 \\
DDD with a disc herniation and predominant axial low & $21(91.3)$ & $2(8.7)$ & \\
$\quad$ back pain (\%) & & & \\
DDD following a discectomy (\%) & $24(82.8)$ & $5(17.2)$ & \\
\hline
\end{tabular}

Bold value indicates significant differences between the groups

${ }^{\mathrm{a}}{ }^{2}$ test

${ }^{\mathrm{b}}$ Independent $t$ test 
General well-being was evaluated using the Short Form36 survey (SF-36) and Oswestry Disability Index (ODI). In both, a score of 0 is equivalent to maximum disability and a score of 100 is equivalent to no disability. Quality of life was assed using the EuroQol-5D (EQ-5D, 0-1, 1 indicating the best health state).

The patient's subjective outcome evaluation was assessed by three questions. The first question was whether they were satisfied with the TDR operation. The second question was whether their current situation was better, the same or worse in comparison with the first 5 years after TDR. The third question was whether they would choose the same surgery again.

\section{Data analysis and statistics}

Baseline patient characteristics were described using mean and standard deviation (SD) and absolute number and percentage. The independent samples $t$ test was used to test for differences in the means of the baseline patient characteristics between patients with or without revision surgery by spinal fusion. Differences in categorical variables in the same groups were tested using the $\chi^{2}$ test.

Kaplan-Meier curves were constructed to assess the cumulative incidence of fusion surgery after TDR over time. Multivariable Cox proportional hazards regression was used to estimate associations between patient characteristics and survival of the TDR. Corresponding hazard ratios (HRs) and $95 \%$ confidence intervals (CIs) were obtained. A multivariable logistic regression model was utilized to identify independent risk factors associated with worse clinical outcome defined as a VAS-score for combined leg and back pain $\geq 5$.0- or an ODI-score $>40$ points at latest follow-up. A cut-off $p$ value of $\leq 0.05$ was considered statistically significant. All analyses were performed using IBM SPSS (Version 23.0).

\section{Results}

\section{Study population}

Altogether, 405 consecutive patients with a TDR were identified. At follow-up, 34 patients had deceased (8.4\%), 14 patients $(3.4 \%)$ refused to participate and 61 patients $(15.1 \%)$ could not be traced. These 109 patients $(26.9 \%)$ were excluded from further analysis. Informed consent was acquired from the remaining 296 patients $(73.1 \%)$ with a mean follow-up of 19.4 years (median 19.3, range $0.2-25.6$ years). A summary of the reasons for exclusion is listed in Fig. 1.

In 59 patients (19.9\%), a revision by spinal fusion with or without removal of the TDR had been performed at a mean of 7.1 years (median 6.7, range 0.2-21.6 years). The mean follow-up after implantation for the 237 patients (80.1\%) without a revision was 19.5 years (median 19.1, range 13.7-25.6 years). Patient characteristics are listed in Table 1. Patients with a TDR at level L2-L3 or multilevel TDR had significantly more risk of needing to undergo revision fusion surgery $(p=0.042$ and $p=0.011$, respectively).

\section{Survival analysis}

A Kaplan-Meier survival curve is depicted in Fig. 2. The vast majority of the fusion procedures $(n=48,81.4 \%)$ occurred in the first 10 years after TDR.

\section{Reoperations}

An overview of all complications requiring a reoperation is provided in Table 2. A total of 108 reoperations were performed in 92 patients, so the overall reoperation rate was $31.1 \%$. Again, the majority occurred in the first 10 years after TDR $(n=81,88.2 \%)$. It was $29.5 \%$ for mono- $(n=69)$ and $39.0 \%$ for the bi-segmental $(n=23)$ TDRs $(p=0.109)$. The mean time until reoperation was 4.0 years (range $0.0-21.6$ years). These were divided into three subgroups: reoperations for immediate device- or technique-related complications $(n=20,6.8 \%)$, reoperations that were related to the surgery in general $(n=7$, $2.4 \%)$ and reoperations for the treatment of persisting symptoms $(n=81,27.4 \%)$.

In 34 patients $(57.6 \%)$, a posterolateral instrumented fusion was performed. In 25 patients (42.4\%) in addition to spinal fusion, the TDR was removed and the intervertebral defect was filled with a femoral head bone strut graft (Fig. 3). In all patients with complaints attributed to ASD, spinal fusion of the index and affected adjacent segment was performed. The remaining 49 reoperations consisted of eight procedures to resolve general complications such as a rectus haematoma or an incisional hernia of the abdominal wall. In 11 patients, the TDR was repositioned after anterior luxation or malposition of the TDR. The remaining 30 procedures were related to radiculopathy or relative spinal stenosis as described by the surgeon. In these patients, a decompression of the hypertrophied ligamentum flavum was performed, often in combination with a laminotomy and undercutting of the facet joints.

To investigate whether learning curve had an impact on reoperation rate, we looked at complications indicative for the latter. In the first 4 years (100 cases), anterior luxation of the TDR occurred in six out the 13 cases $(46.1 \%)$. For malposition, this was two out of six cases (33.3\%). 
Fig. 1 Reasons for exclusion
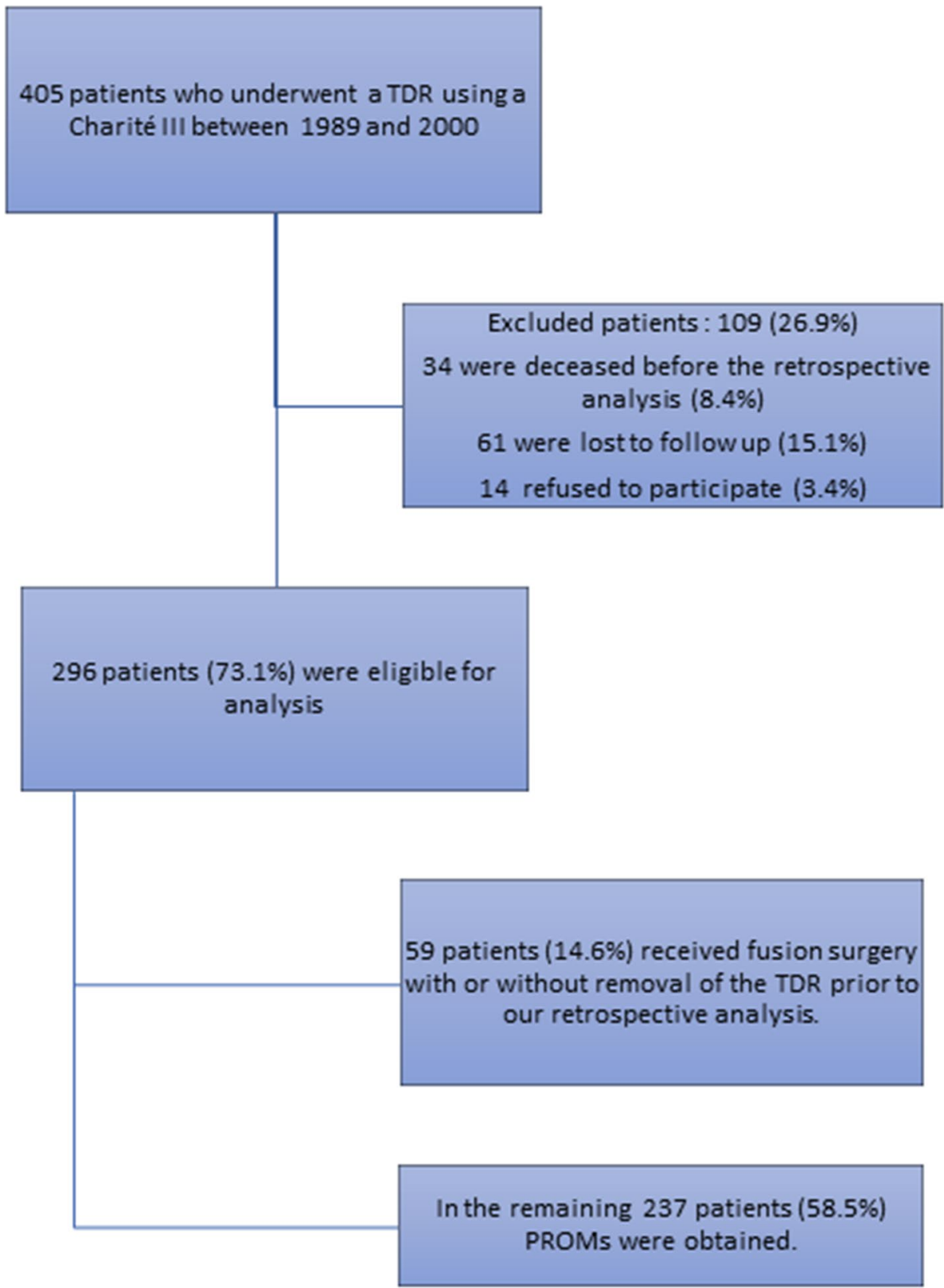

\section{Risk factors for TDR survival and worse clinical outcome}

An overview of all potential risk factors for revision surgery is provided in Table 3. A BMI $>30$ was associated with a significantly lower probability of revision surgery (HR $0.27, p=0.026$ ). Although not statistically significant, previous spinal surgery (discectomy or small decompression) before TDR increased the probability of revision spinal fusion ( $\mathrm{HR}=1.66, p=0.086$ ). In contrast to the univariable analysis (Table 1), no significant differences were seen in the multivariable (adjusted) analysis for multilevel TDR or the level of placement. Therefore, they are not independent risk factors for failure. We tested for associations between all potential risk factors in relation to the different complications requiring a reoperation. A significant association was found for previous spinal surgery and ASD (occurrence of $4 \%$ vs. $14.3 \%, p=0.004)$.

An overview of all potential risk factors for a poor clinical outcome is provided in Table 4. Rheumatoid arthritis (RA) and age at the time of surgery $<45$ years were both associated with a higher probability of a VAS $\geq 5.0$ (OR 5.08 and 1.95 , respectively). Previous spinal surgery, osteoporosis (defined as taking medication for this condition, no data on bone mineral density were available), BMI $>30$ and RA were all associated with a higher probability of an ODIscore $\geq 40$ (OR 2.19, 4.03, 2.40 and 7.15, respectively). The potential risk factors of a TDR at L2-L3 or L3-L4 were not 


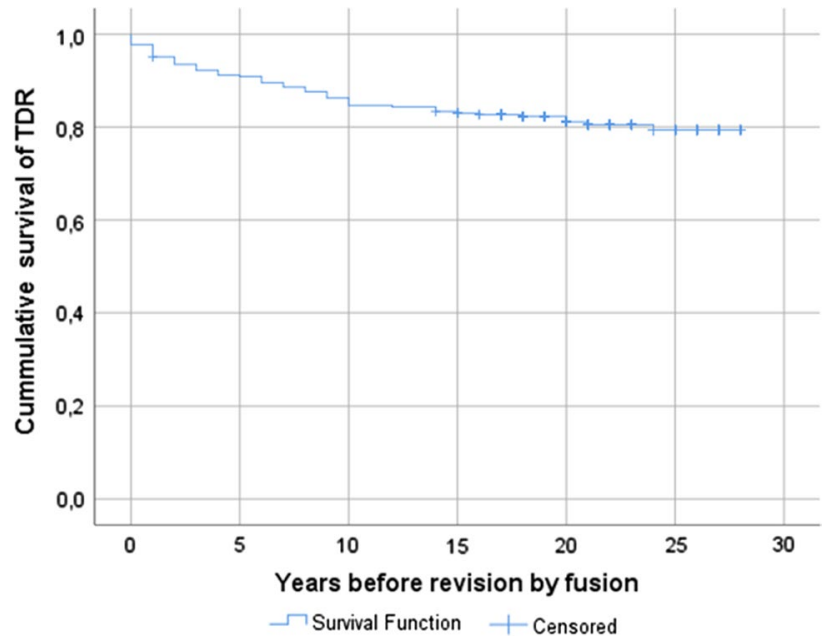

Fig. 2 Kaplan-Meier (survival of the TDR with fusion as endpoint $n=59$ )

included in this multivariable analysis because of too few events to estimate the OR.

When we define failure as a VAS $\geq 5.0$ and/or a revision operation $(n=144,48.6 \%)$, both RA (OR 5.01, $p=0.014)$ and age at the time of surgery $<45$ years (OR $3.10, p=0.001$ ) were associated with a higher probability of failure.

\section{Clinical and subjective outcome evaluation}

Analysis of the clinical parameters, at latest follow-up, showed mean scores for: SF-36 physical of $39.9(n=212$, 12.1 SD), SF-36 mental of $50.9(n=212,11.2 \mathrm{SD})$, VAS-leg of $2.1(n=235,2.8 \mathrm{SD})$, VAS-back of $3.4(n=235,2.9$ SD), ODI $26.7(n=26.7,18.7$ SD) and for EQ 5D of 0.737 $(n=225,0.232 \mathrm{SD})$.

In total, $79.6 \%$ of all patients $(n=235)$ were satisfied with the outcome of their TDR at latest follow-up: 173 patients (73.0\%) would be willing to undergo the same surgery again, 29 patients (12.2\%) were not sure, and 33 patients (13.9\%) would not be willing. When asked to compare their current situation with the first 5 years after surgery, 60 patients (25.3\%) reported a deteriorated clinical outcome.

\section{Discussion}

This study reports the long-term clinical follow-up of the Charité III lumbar TDR implanted by a single surgeon for the treatment of symptomatic DDD. In our study, the overall reoperation rate was $31.1 \%$. In 59 patients $(19.9 \%$ ), revision spinal fusion at the index level was performed. RA, osteoporosis, age at the time of surgery $<45, \mathrm{BMI}>30$ and previous spinal surgery were all associated with a poor clinical outcome.

There is still much debate concerning the use of TDR in terms of fear of deteriorating effect and high rates of late revision operations $[15,18]$. To assess this issue, the accumulation and analysis of long-term data are paramount. There are few studies with a minimal follow-up of 10 years [11-14, 16, 17]. They report a reoperation rate between 5 and $33 \%$. Most of these studies have a mean follow-up around 11 years. In our study, with a mean follow-up of 19.4 years, the vast majority of reoperations, including revision spinal fusions, occurred in the first 10 years after TDR
Table 2 Different indications for a reoperation

\begin{tabular}{lll}
\hline & $N(\%$ of total) & $\begin{array}{l}\text { Mean time in months } \\
\text { till complication } \\
\text { (range) }\end{array}$ \\
\hline Immediate device- or technique-related complications & $20(6.8)$ & $12.87(0.23-128.4)$ \\
Persistent leakage of liquor & 1 & \\
Anterior luxation TDR & 13 & \\
Malposition TDR & 6 & $8.00(0.03-18.4)$ \\
Reoperations that were general surgery related & $7(2.4)$ & \\
Deep surgical site infection & 1 & \\
Ileus requiring hemicolectomy & 1 & $75.84(0.16-258.8)$ \\
Rectus haematoma & 3 & \\
Fascia defect & 2 & \\
Reoperations for the treatment of persisting symptoms CLBP & $81(27.4)$ & \\
Radiculopathy or relative spinal stenosis & 37 & \\
Facet joint arthropathy & 14 & \\
ASD (cranial or caudal) & 15 & \\
Subsidence of the TDR & 15 & \\
Overall complication rate & $92(31.1 \%)$ & \\
\hline
\end{tabular}


Fig. 3 Example case before and after revision of the TDR by posterolateral instrumented spinal fusion and after second stage removal of the TDR, because of persisting complaints
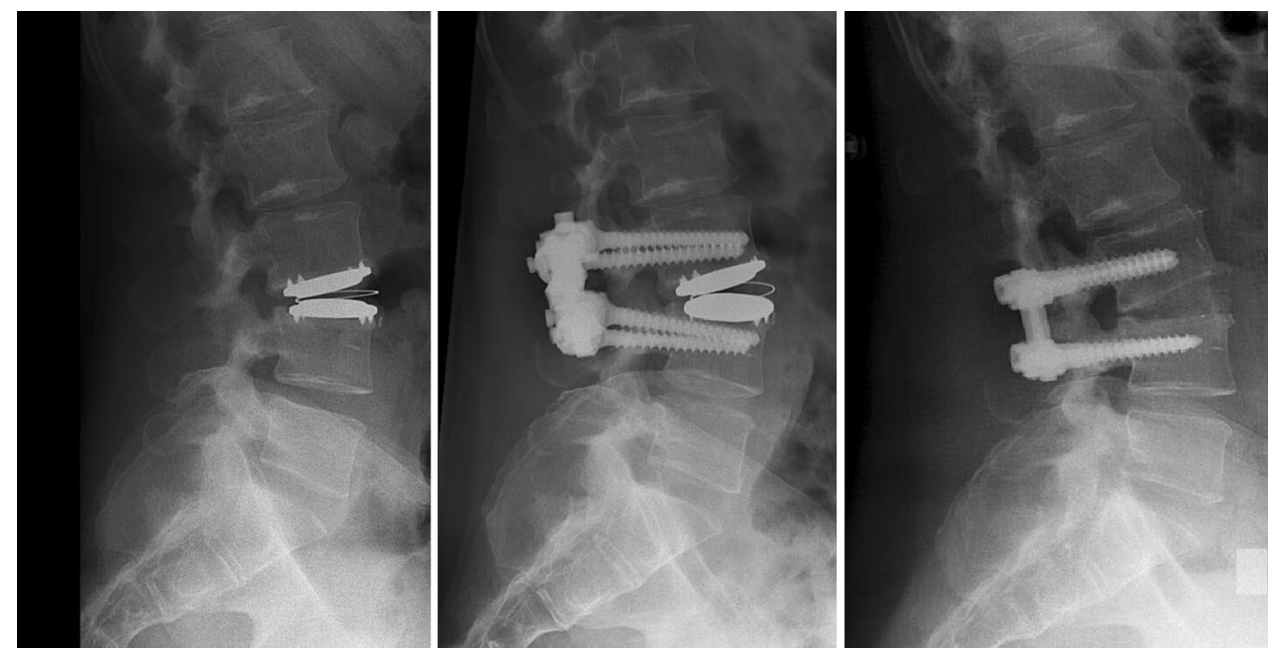

Table 3 Potential risk factors for revision surgery by spinal fusion

\begin{tabular}{|c|c|c|c|c|c|c|}
\hline & \multicolumn{6}{|c|}{ Revision surgery by spinal fusion } \\
\hline & $N$ & No & Yes & Hazard ratio & Confidence interval & $p$ value $^{\mathrm{a}}$ \\
\hline Total population & 296 & $237(80.1)$ & $59(19.9)$ & & & \\
\hline$\geq$ Two levels & 296 & $44(73.3)$ & $16(26.7)$ & 1.48 & $0.82-2.67$ & 0.194 \\
\hline Level L2-L3 or L3-L4 & 296 & $15(68.2)$ & $7(31.8)$ & 1.81 & $0.82-3.99$ & 0.144 \\
\hline Level L4-L5 & 296 & $120(77.9)$ & $34(22.1)$ & 1.19 & $0.71-2.01$ & 0.509 \\
\hline Level L5-S1 & 296 & $146(79.8)$ & $37(20.2)$ & 1.09 & $0.64-1.86$ & 0.760 \\
\hline Male gender & 296 & $102(84.3)$ & $19(15.7)$ & 1.42 & $0.81-2.50$ & 0.219 \\
\hline Age $<45$ years at time of surgery & 296 & $159(77.9)$ & $45(22.1)$ & 1.53 & $0.85-2.76$ & 0.156 \\
\hline Previous spinal surgery & 283 & $40(71.4)$ & $16(28.6)$ & 1.66 & $0.93-2.30$ & 0.086 \\
\hline Smoking & 286 & $81(81.8)$ & $18(18.2)$ & 1.15 & $0.64-2.08$ & 0.633 \\
\hline Rheumatoid arthritis & 294 & $16(76.2)$ & $5(23.8)$ & 1.30 & $0.52-3.25$ & 0.582 \\
\hline COPD & 294 & $17(81.0)$ & $4(19.0)$ & 0.75 & $0.24-2.41$ & 0.634 \\
\hline Osteoporosis $^{\mathrm{b}}$ & 293 & $15(71.4)$ & $6(28.6)$ & 1.79 & $0.76-4.17$ & 0.181 \\
\hline $\mathrm{BMI}>30$ & 289 & $48(92.3)$ & $4(7.7)$ & 0.27 & $0.08-0.86$ & 0.026 \\
\hline
\end{tabular}

Bold value indicates significant differences between the groups

${ }^{\mathrm{a} C o x}$ multivariate regression

${ }^{b}$ Defined as taking medication for the treatment of osteoporosis

( $88.2 \%$ and $81.4 \%$, respectively). It appears that there is no need for fear of late revision operations according to our data.

Our overall reoperation rate of $31.1 \%$ was similar to the rate of 33\% in the study of Laugesen et al. [16] but higher in comparison with other long-term follow-up studies [11-14, $16,17]$ reporting incidences between 5 and $9 \%$. The mean follow-up in our study is higher in comparison with those studies [11-14, 16, 17], which may account for a relatively higher incidence of reoperations. Furthermore, this difference may have been caused by sub-optimal patient selection for the index TDR surgery. In later years, improvements have been made in terms of surgical technique, imaging of the spine and more appropriate patient selection based on social profile $[11,15]$. This might explain the tendency of earlier studies, or those with longer follow-up, to report less favourable outcomes than those of more recent studies [16]. Learning curve does not seem to be a factor, since complications indicative for the latter such as anterior luxation or malposition of the TDR are evenly distributed over the years.

TDR is associated with progression of facet joint arthropathy (FJA) at the index level [10]. In our study, in 14 out of 59 patients (23.7\%), FJA was reported as the reason for revision spinal fusion. However, it is likely that in patients classified as suffering from ASD, spinal stenosis or subsidence, FJA has been a factor as well. This assumption is supported by the observation that in all patients $(n=15)$ with revision spinal surgery for ASD, the index level was also fused. 
Table 4 Overview of potential risk factors for a poor clinical outcome

\begin{tabular}{|c|c|c|c|c|c|c|c|c|}
\hline & \multicolumn{4}{|l|}{ VAS-score } & \multicolumn{4}{|l|}{ ODI-score } \\
\hline & $N<5.0(\%)$ & $N \geq 5.0(\%)$ & Odds ratio $(\mathrm{CI})$ & $p$ value $^{\mathrm{a}}$ & $N<40(\%)$ & $N \geq 40(\%)$ & Odds ratio $(\mathrm{CI})$ & $p$ value $^{\mathrm{a}}$ \\
\hline Total population & $161(61.7)$ & $100(38.3)$ & & & $155(69.5)$ & $68(30.5)$ & & \\
\hline$\geq$ Two levels & $29(54.7)$ & $24(45.3)$ & $1.08(0.28-4.15)$ & 0.909 & $29(63.0)$ & $17(37.0)$ & $1.20(0.27-5.45)$ & 0.813 \\
\hline Level L4-L5 & $76(44.1)$ & $76(55.9)$ & $1.48(0.40-5.46)$ & 0.557 & $80(66.7)$ & $40(33.3)$ & $1.26(0.29-5.50)$ & 0.762 \\
\hline Level L5-S1 & $104(64.2)$ & $58(35.8)$ & $0.98(0.28-3.42)$ & 0.969 & $93(69.9)$ & $40(30.1)$ & $1.05(0.25-4.33)$ & 0.950 \\
\hline Male gender & $71(65.1)$ & $38(34.9)$ & $1.19(0.68-2.08)$ & 0.552 & $73(78.5)$ & $20(21.5)$ & $1.84(0.93-3.65)$ & 0.079 \\
\hline Age $<45$ years at time of surgery & $95(57.2)$ & $71(42.8)$ & $1.95(1.06-3.56)$ & $\mathbf{0 . 0 3 1}$ & $98(69.5)$ & $43(30.5)$ & $1.38(0.69-2.77)$ & 0.362 \\
\hline Previous spinal surgery & $23(48.9 \%)$ & $24(51.1 \%)$ & $1.80(0.90-3.62)$ & 0.098 & $24(55.8)$ & $19(44.2)$ & $2.19(1.02-4.72)$ & 0.046 \\
\hline Smoking & $48(53.9)$ & $41(46.1)$ & $1.55(0.87-2.75)$ & 0.135 & $42(60.9)$ & $27(39.1)$ & $1.95(0.98-3.85)$ & 0.056 \\
\hline Rheumatoid arthritis & $6(31.6)$ & $13(68.4)$ & $5.08(1.54-16.7)$ & 0.008 & $5(29.4)$ & $12(70.6)$ & 7.15 (1.87-27.4) & 0.004 \\
\hline COPD & $9(45.0)$ & $11(55.0 \%)$ & $1.67(0.54-5.16)$ & 0.370 & $9(56.3)$ & $7(43.8)$ & $0.72(0.18-2.84)$ & 0.722 \\
\hline Osteoporosis ${ }^{\mathrm{b}}$ & $8(40.0)$ & $12(60.0 \%)$ & $1.20(0.37-3.89)$ & 0.763 & $5(31.3)$ & $11(68.8)$ & $\mathbf{4 . 0 3}(1.01-16.0)$ & 0.048 \\
\hline $\mathrm{BMI}>30$ & $27(54.0)$ & $23(46.0 \%)$ & $1.47(0.75-2.87)$ & 0.266 & $23(56.1)$ & $18(43.9)$ & $2.40(1.10-5.26)$ & 0.029 \\
\hline
\end{tabular}

Bold value indicates significant differences between the groups

${ }^{a}$ Multivariable logistic regression

${ }^{\mathrm{b}}$ Defined as taking medication for the treatment of osteoporosis

A prospective study of Siepe et al. [15] with 181 patients and a mean follow-up of 7.4 years reported a reoperation rate of $16 \%$. The incidence of either general surgery- or devicerelated complications in that study amounted to $7.2 \%$ and is quite similar to the $9.2 \%$ in the current study. It must be noted that in that study, as in the RCTs comparing TDR with fusion [1-3], numerous exclusion criteria such as previous spinal surgery, obesity and chronic steroid use were applied. As that was not the case in the current study, our population might be a better representation of patients with DDD in daily clinical practice.

In addition, the reoperation rates should similarly be compared with the rates that have been published on lumbar fusions. A large retrospective cohort study in adults who underwent lumbar fusion for degenerative spine disorders between 1990 and $1993(n=24.882)$ showed a cumulative incidence of reoperations of $21.5 \%$ after 11-year follow-up [20]. This is similar to the revision fusion rate in our study, but our overall reoperation rate is higher. However, when we look at our reoperation rate after 11-year follow-up (27.7\%, $n=82$ ), the difference in reoperation rate is less pronounced.

\section{Risk factors for survival and worse clinical outcome}

A BMI $>30$ was associated with a significantly lower probability of revision surgery by spinal fusion (HR 0.27). It is possible that the surgeons were less inclined to perform revision surgery because of associated higher complication rates in the obese patients [21]. Moreover, it is possible that these obese patients had a lower activity resulting in less wear of the TDR, as has been published for obese patients with hip and total knee replacements [22]. The fact that in our population, $\mathrm{BMI}>30$ was associated with an ODI-score $>40$ might be indicative of the latter. However, not statistically significant $(p=0.086)$, previous spinal surgery before TDR tended to increase the probability of revision spinal fusion $(\mathrm{HR}=1.66)$ in our population.

In contrast to the study of Siepe et al. [15], multilevel TDR did not significantly increase the probability of revision surgery or poor clinical outcome in our multivariable analysis. These findings are consistent with several other studies $[16,19,23]$. The level of TDR was not associated with an increase in revision surgery or a decrease in clinical outcome as well. This again is consistent with the literature [24, 25].

Rheumatoid arthritis, osteoporosis, age at the time of surgery $<45$ and previous spinal surgery were all associated with a VAS-score $\geq 5.0$ or an ODI-score $\geq 40$. Tropiano et al. [19] showed similar results for age and previous spinal surgery.

\section{Clinical and subjective outcome evaluation}

PROMs were obtained at latest follow-up in all patients without a previous revision by spinal fusion. This means that these outcome measurements are not a reflection of our total population. Furthermore, no preoperative questionnaires were available for comparison. However, it is possible to compare our outcome measurement with those reported in the other mid- to long-term follow-up studies on TDR.

Siepe et al. [15], Lu et al. [13] and Park et al. [17] reported at latest follow-up an ODI-score of 20.3, 13.2 and 22.4 and a VAS-score of 3.3, 1.5 and 3.4, respectively. These numbers are comparable to those in our study (VAS-score 
of 3.4, ODI-score of 26.7) although it must be noted that in these three studies, patients with a revision by spinal fusion were included (11\%, 6.3\% and 9.3\%, respectively). Laugesen et al. [16] reported a VAS-score of 2.4 and a SF-36 physical of 31.9 in their group without a revision, both similar to our population (SF-36 physical of 39.9).

In our study, $79.6 \%$ of the patients were satisfied with their TDR and $73.0 \%$ would be willing to undergo the same surgery again for similar complaints. When assuming that all patients with a fusion were not satisfied and not willing, these percentages would drop to $63.6 \%$ and $58.16 \%$, respectively. Previously mentioned studies reported percentages between $86.3-64.9 \%$ and $79.3-52.6 \%$, respectively [13, 15-17]. To our opinion, the clinical status of patients after TDR at a follow-up of almost 20 years is not substantially different from those at 8-12 years follow-up [13, 15-17].

\section{Limitations and strengths}

The current study's main limitation is its retrospective nature. PROMs were obtained at latest follow-up, and no preoperative questionnaires were available for comparison. Unfortunately, most of the other long-term follow-up studies had a retrospective design [11, 12, 14, 17]. A lack of control group (either non-operative or index spinal fusion) can be attributed to all these studies.

The number of patients included in any study has a vital influence on the outcome and whether a study is representative or not. We included a total of 296 patients. Our mean follow-up of 19.4 years is the longest available in the literature. Despite our long follow-up, only $18.5 \%$ of our patients were lost to follow-up or refused to participate. Consequently, there is a high chance of generalizability of our study.

The results presented in this study demonstrate a revision spinal fusion rate of $19.9 \%$ after a mean follow-up of 19.4 years. Fear of excessive late complications or reoperations following the primary TDR cannot be substantiated since the vast majority of all reoperations occurred in the first 10 years after TDR. Proper patient selection considering the identified risk factors for worse outcome may help to improve the clinical and functional outcome in these patients.

Open Access This article is licensed under a Creative Commons Attribution 4.0 International License, which permits use, sharing, adaptation, distribution and reproduction in any medium or format, as long as you give appropriate credit to the original author(s) and the source, provide a link to the Creative Commons licence, and indicate if changes were made. The images or other third party material in this article are included in the article's Creative Commons licence, unless indicated otherwise in a credit line to the material. If material is not included in the article's Creative Commons licence and your intended use is not permitted by statutory regulation or exceeds the permitted use, you will need to obtain permission directly from the copyright holder. To view a copy of this licence, visit http://creativecommons.org/licenses/by/4.0/.

\section{References}

1. Guyer RD et al (2009) Prospective, randomized, multicenter Food and Drug Administration investigational device exemption study of lumbar total disc replacement with the CHARITE artificial disc versus lumbar fusion: five-year follow-up. Spine J 9(5):374-386

2. Zigler JE, Glenn J, Delamarter RB (2012) Five-year adjacent-level degenerative changes in patients with single-level disease treated using lumbar total disc replacement with ProDisc-L versus circumferential fusion. J Neurosurg Spine 17(6):504-511

3. Skold C, Tropp H, Berg S (2013) Five-year follow-up of total disc replacement compared to fusion: a randomized controlled trial. Eur Spine J 22(10):2288-2295

4. Ha KY, Lee JS, Kim KW (2008) Degeneration of sacroiliac joint after instrumented lumbar or lumbosacral fusion: a prospective cohort study over five-year follow-up. Spine (Phila Pa 1976) 33(11):1192-1198

5. Kumar MN, Jacquot F, Hall H (2001) Long-term follow-up of functional outcomes and radiographic changes at adjacent levels following lumbar spine fusion for degenerative disc disease. Eur Spine $\mathbf{J}$ 10(4):309-313

6. Lee CK (1988) Accelerated degeneration of the segment adjacent to a lumbar fusion. Spine (Phila Pa 1976) 13(3):375-377

7. Park Y et al (2011) Cranial facet joint violations by percutaneously placed pedicle screws adjacent to a minimally invasive lumbar spinal fusion. Spine J 11(4):295-302

8. van den Eerenbeemt KD et al (2010) Total disc replacement surgery for symptomatic degenerative lumbar disc disease: a systematic review of the literature. Eur Spine J 19(8):1262-1280

9. Jacobs $\mathrm{W}$ et al (2012) Total disc replacement for chronic back pain in the presence of disc degeneration. Cochrane Database Syst Rev 2012(9):CD008326

10. Siepe CJ et al (2010) The fate of facet joint and adjacent level disc degeneration following total lumbar disc replacement: a prospective clinical, X-ray, and magnetic resonance imaging investigation. Spine (Phila Pa 1976) 35(22):1991-2003

11. David T (2007) Long-term results of one-level lumbar arthroplasty: minimum 10-year follow-up of the CHARITE artificial disc in 106 patients. Spine (Phila Pa 1976) 32(6):661-666

12. Lemaire JP et al (2005) Clinical and radiological outcomes with the Charite artificial disc: a 10-year minimum follow-up. J Spinal Disord Tech 18(4):353-359

13. Lu SB et al (2015) An 11-year minimum follow-up of the Charite III lumbar disc replacement for the treatment of symptomatic degenerative disc disease. Eur Spine J 24(9):2056-2064

14. Putzier M et al (2006) Charite total disc replacement-clinical and radiographical results after an average follow-up of 17 years. Eur Spine J 15(2):183-195

15. Siepe CJ et al (2014) Mid- to long-term results of total lumbar disc replacement: a prospective analysis with 5- to 10-year follow-up. Spine J 14(8):1417-1431

16. Laugesen LA et al (2017) Patient-reported outcomes and revision rates at a mean follow-up of 10 years after lumbar total disc replacement. Spine (Phila Pa 1976) 42(21):1657-1663

17. Park SJ et al (2016) Long-term outcomes following lumbar total disc replacement using ProDisc-II: average 10-year follow-up at a single institute. Spine (Phila Pa 1976) 41(11):971-977

18. Ding F et al (2017) Total disc replacement versus fusion for lumbar degenerative disc disease: a systematic review of overlapping metaanalyses. Eur Spine J 26(3):806-815 
19. Tropiano $P$ et al (2005) Lumbar total disc replacement. Seven to eleven-year follow-up. J Bone Joint Surg Am 87(3):490-496

20. Martin BI et al (2007) Reoperation rates following lumbar spine surgery and the influence of spinal fusion procedures. Spine (Phila $\mathrm{Pa}$ 1976) 32(3):382-387

21. Patel $\mathrm{N}$ et al (2007) Obesity and spine surgery: relation to perioperative complications. J Neurosurg Spine 6(4):291-297

22. McClung CD et al (2000) Relationship between body mass index and activity in hip or knee arthroplasty patients. J Orthop Res 18(1):35-39

23. Di Silvestre M et al (2009) Two-level total lumbar disc replacement. Eur Spine J 18(Suppl 1):64-70
24. Siepe CJ et al (2007) Total lumbar disc replacement: different results for different levels. Spine (Phila Pa 1976) 32(7):782-790

25. Sinigaglia R et al (2009) Comparison of single-level L4-L5 versus L5-S1 lumbar disc replacement: results and prognostic factors. Eur Spine J 18(Suppl 1):52-63

Publisher's Note Springer Nature remains neutral with regard to jurisdictional claims in published maps and institutional affiliations.

\section{Affiliations}

\section{J. Kitzen ${ }^{1}$ - M. G. M. Schotanus ${ }^{2}$ - S. M. J. van Kuijk ${ }^{3}$ - E. M. C. Jutten ${ }^{1}$ - N. P. Kort ${ }^{2}$ - L. W. van Rhijn ${ }^{1}$ P. C. Willems ${ }^{1}$}

1 Department of Orthopedic Surgery, Maastricht University Medical Centre, P.O. Box 5800, 6202 AZ Maastricht, The Netherlands

2 Department of Orthopedic Surgery, Zuyderland Medical Centre, P.O. Box 500, 6130 MB Sittard-Geleen,

The Netherlands
3 Department of Clinical Epidemiology, Maastricht University Medical Centre, P.O. Box 5800, 6202 AZ Maastricht, The Netherlands 